1 Title page

2 The EXO70B2 exocyst subunit contributes to papillae and encasement formation in anti-fungal

3 defence in Arabidopsis

4 Jitka Ortmannová ${ }^{1,2}$, Juraj Sekereš1, Ivan Kulich² ${ }^{2}$ Jiří Šantrůček ${ }^{3}$, Petre Dobrev ${ }^{4}$, Viktor Žárský1,2, Tamara Pečenková $^{1 *}$

Laboratory of Cell Biology, Institute of Experimental Botany, Czech Academy of Sciences, Rozvojová 263, 165 02, Prague 6, Czech Republic

Laboratory of Cell Morphogenesis, Department of Experimental Plant Biology, Charles University in Prague, Faculty of Science, Viničná 5, 128 44, Prague 2, Czech Republic

Laboratory of Applied Proteomics, University of Chemistry and Technology, Prague, Technická 5, 16628, Prague 6, Czech Republic

Laboratory of Hormonal Regulations in Plants, Institute of Experimental Botany, Czech Academy of Sciences, Rozvojová 263 , 165 02, Prague 6, Czech Republic

authors email addresses: jitka.ortmann@gmail.com, juro.seky@gmail.com, ikulich@gmail.com, jiri.santrucek@vscht.cz, dobrev@ueb.cas.cz, viktor@natur.cuni.cz, pecenkova@ueb.cas.cz

* Correspondence: Tamara Pečenková; pecenkova@ueb.cas.cz; (+420225106458)

16 date of submission: June $18^{\text {th }}, 2021$

17 number of tables: 0

18 number of figures: 7

19 the word count (start of the introduction to the end of the acknowledgements, excluding materials and methods): 3866

20 supplementary figures: 9

21 supplementary tables: 2

22 Running title: Exocyst shapes papillae and haustorial encasements 


\section{Highlight (30)}

The exocyst complex containing EXO70B2 subunit has specific role in papilla and encasement formation, implemented in coordination with the pathway regulated by the SYP121 SNARE complex in defence.

Abstract (200)

In the reaction to non-adapted Blumeria graminis f. sp. hordei (Bg), Arabidopsis thaliana leaf epidermal cells deposit cell wall reinforcements called papillae or seal fungal haustoria in encasements, both of which involve intensive exocytosis. A plant syntaxin SYP121/PEN1 has been found to be of key importance for the timely formation of papillae, and the vesicle tethering complex exocyst subunit EXO70B2 has been found to contribute to their morphology. Here, we identify a specific role for the EXO70B2-containing exocyst complex in the papillae membrane domains important for the callose deposition and GFP-SYP121 delivery to the focal attack sites, as well as its contribution to encasement formation. The mRuby2-EXO70B2 co-localises with the exocyst core subunit SEC6 and GFP-SYP121 in the membrane domain of papillae, and both proteins have the capacity to directly interact. The exo70B2/syp 121 double mutant has a reduced number of papillae and haustorial encasements in response to $\mathrm{Bg}$, indicating an additive role of the exocyst in SYP121 coordinated non-host resistance. In summary, we report cooperation between the plant exocyst and a SNARE protein in penetration resistance against non-adapted fungal pathogens.

\section{Keywords and abbreviations}

penetration 1; exocyst 2; secretion 3, nonhost resistance 4, SNARE 5, callose 6

$\begin{array}{ll}\text { Bg } & \text { Blumeria graminis f. sp. hordei } \\ \text { PAMP } & \text { pathogen associated molecular patterns } \\ \text { ROS } & \text { reactive oxygen species } \\ \text { EHM } & \text { extra haustorial membrane } \\ \text { Ep } & \text { Erysiphe pisi } \\ \text { PM } & \text { plasma membrane } \\ \text { SNARE } & \text { soluble N-ethylmaleimide-sensitive factor Attachment protein REceptors } \\ & \\ \text { SNAP } & \text { soluble N-ethylmaleimide-sensitive factor adaptor protein } \\ \text { VAMP } & \text { vesicle-associated membrane protein } \\ \text { MVB } & \text { multivesicular bodies } \\ \text { hpi } & \text { hour post inoculation } \\ \text { KO } & \text { knockout line } \\ \text { HSD } & \text { honestly significant difference } \\ \text { SA } & \text { salicylic acid } \\ \text { BD } & \text { binding domain } \\ \text { AD } & \text { activation domain } \\ \text { NA } & \text { numeric aperture }\end{array}$

\section{Introduction}

One of the main tiers of plant non-host resistance is a secretory pathway. At the moment of detection of a pathogen at the plant surface, pathogen-associated molecular pattern (PAMP)-triggered immunity is activated and plant cells start to react and re-polarize their secretory pathway to the attack sites (Schmelzer, 2002; Jones and Dangl, 2006; Lee et al., 2017). The newly established secretory domain allows the plant cells to deposit focal cell wall reinforcements called papillae (Bestwick et $a l ., 1995)$. Their composition is a mixture of cell wall and antimicrobial components, such as callose (beta-1,3-glucan), pectins, lignins, reactive oxygen species (ROS), phytoalexins, and thionins (Schmelzer, 2002; Chowdhury et al., 2014). Despite the surface barriers, some fungal hyphae may invade plant cells with feeding structures called haustoria. Haustoria are surrounded by a specialized 
extrahaustorial membrane, the origin of which remains elusive (EHM) (Micali et al., 2011). To stop fungal growth, plant cells seal the haustoria by encasements, defensive structures made of similar components to the papillae (Heath and Heath, 1971; Zeyen et al., 2002).

In Arabidopsis thaliana, non-host resistance remains sufficient against evolutionarily non-adapted fungi, such as Erysiphe pisi (Ep) or Bg (Vogel and Somerville, 2000; Lipka et al., 2005; Consonni et al., 2006). A forward genetic screen revealed that the PENETRATION1 (PEN1/SYP121) locus plays a crucial role in this non-host resistance (Collins et al., 2003; Stein et al., 2006; Lipka et al., 2008). SYP121 belongs to the family of plasma membrane (PM) Qa-Soluble N-ethylmaleimide-sensitive factor Attachment protein REceptors (SNAREs). SYP121 forms a ternary complex with the cytoplasmic Qbc-SNARE SNAP33 (soluble N-ethylmaleimide-sensitive factor adaptor protein 33) and the R-SNAREs VAMP721 or VAMP722 (vesicle-associated membrane protein 721/722) (Kwon et al., 2008). This complex promotes the exocytosis of secretory vesicles carrying defence-related cargoes to plant cell/fungus contact sites and mediates papillae formation (Assaad et al., 2004; Kwon et al., 2008; Meyer et al., 2009). GFP-SYP121 also associates in a GNOM-dependent manner with exosomes in the extracellular matrix of both papillae and encasements (Nielsen et al., 2012; Rutter and Innes, 2017). However, the canonical role of SYP121 probably relates to intracellular endomembranes fusions, possibly at the Golgi (Nielsen and Thordal-Christensen, 2012, 2013; Nielsen et al., 2017). The SYP121-interacting R-SNARE VAMP727 has been found to operate on Rab-GTPase ARA7-positive multivesicular bodies (MVB), indicative of the involvement of these MVB in secretion into the papillary matrix (An et al., 2007; Ebine et al., 2011; Nielsen et al., 2012; Heard et al., 2015).

In yeasts and mammals, SNARE proteins execute their function in cooperation with other proteins and protein complexes, one of which is the exocyst complex (Hsu et al., 1996; Sivaram et al., 2005; Dubuke et al., 2015; Yue et al., 2017). The exocyst vesicle tethering complex assembles from the core subunits SEC3 (membrane lipid-interacting subunits), SEC5, SEC6, SEC8, SEC10, SEC15 and EXO84, and the plasma membrane lipid-interacting and localizing subunit EXO70 (TerBush et al., 1996; Guo et al., 1997; Liu et al., 2018; Zhu et al., 2019). While being encoded by a single gene in the yeast and mammalian genome, EXO70 is encoded by many paralogs in angiosperm genomes (Elias et al., 2003; Cvrcková et al., 2012). In accordance, the plant exocyst complex has multiple functions, e. g. seed coat deposition, tip growth, cytokinesis, recycling and autophagy (Cole et al., 2005; Synek et al., 2006; Hála et al., 2008; Fendrych et al., 2010, 2013; Kulich et al., 2010; Drdova et al., 2013). It has been hypothesized that the different isoforms may target the exocyst and exocytosis to different plant cell PM domains even within a single cell type such as a growing pollen tube or trichomes (Žárskỳ et al., 2009, 2013; Sekereš et al., 2017; Synek et al., 2017; Kubátová et al., 2019).

In Arabidopsis, the specific role of the EXO70 isoform EXO70B2 has been documented in PAMPtriggered immunity regulation and papillae biogenesis (Pečenková et al., 2011; Stegmann et al., 2012; Wang et al., 2020). Here, we show that in addition to EXO70B2-GFP, the core exocyst subunit SEC6 tagged with GFP is also localised to papillae and encasement structures indicating that whole exocyst complex is there. Further on, we observed an increase in the penetration rate of the non-adapted powdery mildew fungi $\mathrm{Bg}$ (and also Ep) in two exocyst core subunit mutants as a probable consequence of slower callose deposition. The EXO70B2 and SYP121 have a capacity to directly interact and to mutually affect papilla-related localisations. The encasements formation and callose accumulation are reduced in the double mutant exo70B2/syp 121 . These results indicate that the fully functional exocyst complex involving the isoform EXO70B2 is important for penetration resistance, likely as a component of the previously described SYP121-dependent secretory pathway.

\section{Materials and methods}

Plant material 
The seeds of Arabidopsis thaliana were sterilised and plated on 1/2 MS with vitamins, $1 \%$ sucrose medium. The plants were grown in vitro for 10 days and used for qRT-PCR, confocal imaging, or transferred to Jiffy tablets and grown in a growth chamber under short day conditions $\left(21^{\circ} \mathrm{C}, 10 / 14 \mathrm{~h}\right.$ light/dark, $80 \%$ humidity and light intensity of $125 \mu \mathrm{mol} \mathrm{m}-2 \mathrm{~s}-1$ in a range from $400-700 \mathrm{~nm}$ ).

The mutant lines of Arabidopsis used in this study were as follows: knockout line (KO) sec5a-1 (GABI_731C01, Fig. S6), KO line syp121-1 (Collins et al., 2003), knock-down line sec8-m4 (Cole et al., 2005), exo70B2-2 KO line (Pečenková et al., 2011). The transgenic lines of Arabidopsis that were used in this study were: EXO70B2: EXO70B2-GFP; EXO70B2:GFP-EXO70B2 (this work); SYP121:GFP-SYP121 (Kato et al., 2010); SEC6:SEC6-GFP and 35S:GFP-EXO70A1 (Fendrych et al., 2010); EXO70B1:GFP-EXO70B1 (Kulich et al., 2013).

Plasmid construction and generation of transgenic lines

All constructs (GFP-EXO70B2, EXO70B2-GFP and mRuby2-EXO70B2) were prepared with the Phusion PC (NEB, USA) reaction using as a template either genomic DNA for intron-less genes and promoters or cDNA. A list of the primers used is presented in Supplementary Table 1, as well as the lengths of the amplified fragments, restriction sites used for the cloning procedure and destination vectors used in the cloning procedure. The sequenced vectors were used to transform the Agrobacterium tumefaciens GV3101 strain. Arabidopsis WT or respective mutants were transformed by the Agrobacterium-mediated floral dip method (Clough and Bent, 1998).

Pathogen inoculation and cytology

For the pathogen inoculation experiments, plants were cultivated under short day conditions $\left(21^{\circ} \mathrm{C}\right.$, $10 / 14 \mathrm{~h}, 75 \%$ humidity with a light intensity of $125 \mu \mathrm{mol} \mathrm{m}-2 \mathrm{~s}-1)$. Bg was cultivated continuously on fresh barley (Golden promise) grown under short day conditions $\left(19^{\circ} \mathrm{C}, 10 / 14 \mathrm{~h}, 50 \%\right.$ humidity, and a light intensity of $70 \mu \mathrm{mol} \mathrm{m}-2 \mathrm{~s}-1$ ). Ep was cultivated continuously on fresh pea (Pisum sativum variety Petit provencal) under short day conditions $\left(19^{\circ} \mathrm{C}, 10 / 14 \mathrm{~h}, 60 \%\right.$ humidity and light intensity of $70 \mu \mathrm{mol} \mathrm{m}-2 \mathrm{~s}-1)$. The Blumeria graminis $f$. sp. hordei isolate A6 on barley genotype P01 was kindly provided by Lenka Burketová, Laboratory of Pathological Plant Physiology in Prague, IEB AS, Czech Republic. Paul Schulze-Lefert from the Max Planck Institute for Plant Breeding Research, Cologne, kindly provided the Erysiphe pisi isolate. The plants, approximately 4 weeks old, were inoculated by spreading spores from infected barley or pea onto the adaxial site of their leaves (from leaf to leaf). The 5th and 6th leaves were cut off at selected hpi and cleared with $96 \%$ ethanol or chloral hydrate. For callose visualisation, cleared leaves were stained with an alkali solution of aniline blue (Eschrich and Currier, 1964). For penetration rate visualisation, fungal structures were stained with $250 \mathrm{mg} / \mathrm{ml}$ trypan blue in lactophenol/ethanol solution (Vogel and Somerville, 2000). For quantification purposes, we considered only epidermal cells that were attacked by a single germinated spore. Stained leaves were observed with classical epifluorescence microscopy or optical microscopy using a Nikon Eclipse TE 2000-E inverted microscope. For the bacterial sensitivity assay, the Arabidopsis seedling flood-inoculation assay with Pseudomonas syringae pv. tomato DC3000 was utilized according to (Ishiga et al., 2011) with minor modifications.

Transcript detection and semiquantitative RT-PCR

RNA was isolated from 100-120 mg of 14-day-old plants using the RNeasy kit (Qiagen). For cDNA synthesis, $2.5 \mu \mathrm{g}$ of isolated mRNA was used. For PCR amplification, $2.5 \mu \mathrm{L}$ of $20 \times$ diluted cDNA $(2 \mu \mathrm{g} / \mu \mathrm{l})$ was used, and the gene-specific pairs of primers were used for semiquantitative PCR (Supplementary Table S1).

Microscopy 
Microscopic observation of Arabidopsis plantlets attacked with pathogens was performed using an inverted spinning disk confocal microscope with a high-resolution camera (Yokogawa CSU-X1 on Nikon Ti-E platform, laser box Agilent MLC400, with sCMOS camera Andor Zyla CSU-X1). The dynamic study was performed with a high-speed camera (with sCMOS Andor iXon DU-897) using filter cubes for GFP. Nikon Plan Apochromat x60 WI (NA = 1.2) and Plan Apochromat x100 OI (NA $=1.45$ ) objective lenses were used for imaging with 488 and $561 \mathrm{~nm}$ laser lines. The exposure time was $700 \mathrm{~ms}$, with a $488 \mathrm{~nm}$ laser power of $75 \%$. Fluorescence profiles and acquired images were exported from NIS ELEMENTS 4.1 software (Nikon, Tokyo, Japan); identical settings were used for each image. Images were then analysed using ImageJ Fiji software (http://rsbweb.nih.gov/ij/) and assembled with the freeware Inkscape programme into figures. Microscopic analysis of tissue staining with aniline or trypan blue was performed using an Olympus BX51 microscope with an attached DP50 camera x100 OI (NA = 1.35) objective (Olympus; Trypan blue) or Zeiss AxioImager ApoTome 2 microscope 20x objective (Aniline blue). For the analysis of callotic spots, the tile images obtained with the Zeiss AxioImager ApoTome2 microscope under a 20x objective were analysed using Fiji software using the parameters of circularity of 0.5 and particle size from 200-1500 px. To exclude spots from non-specific precipitates of aniline blue in dead cells from the analysis, the cell state was assessed under bright field prior to quantification. Images obtained using the Olympus camera were analysed with analysis software and those obtained using Zeiss microscopes were analysed with ZEISS ZEN BLUE software. To perform lambda scans, a Zeiss LSM 880 confocal scanning microscope with a Zeiss C-Apochromat 40x (NA = 1.2) W Korr FCS M27 or C-Apochromat x63 OI $(\mathrm{NA}=1.45)$ objective was used. For visualising the fungal structure in vivo, propidium iodide PI 1:500 or FM4-64 dye 1:1000 diluted in water was used. The excitation wavelengths were $488 \mathrm{~nm}$ for GFP and $561 \mathrm{~nm}$ for mRuby2, PI and FM4-64. Linear unmixing was performed with ZEISS ZEN BLACK software.

\section{Yeast two-hybrid assay}

The SYP121 DNA (obtained from Riken) was amplified from cDNA with primers excluding the transmembrane domain and cloned into the pGADT7 vector (Clontech Laboratories, Inc.). All other exocyst constructs used in the study have been previously described [38,48,73](Hála et al., 2008; Pečenková et al., 2011; Vukašinović et al., 2014). Different pGBKTs with pGAD with an inserted non-coding piece of the pENTR3C vector were used as negative controls. At least 10 positive colonies from -Leu/-Trp plates were resuspended in $150 \mu \mathrm{l}$ of sterile water, diluted 30x and 900x, and subsequently plated on $-\mathrm{Leu} /-\mathrm{Tr}$ /-His/-Ade plates.

\section{Protein extraction, SDS gel electrophoresis and Western blotting}

Total protein extracts were isolated from 2-4-week-old plants transformed with EXO70B2-GFP at different time points ranging from 0-24 hpi with $\mathrm{Bg}$ or water (mock). The protein extraction Sec6/8 buffer adjusted for exocyst extraction was used (20 mM HEPES, pH 6.8, $150 \mathrm{mM} \mathrm{NaCl}, 1 \mathrm{mM}$ EDTA, $1 \mathrm{mM}$ DTT, and 0.5\% Tween, supplemented with protease inhibitor cocktail (SigmaAldrich)). After one hour of lysis, the extracts were spun down, and the supernatants were boiled in $6 \mathrm{x}$ SDS loading buffer. The proteins were loaded for $10 \%$ SDS-PAGE and blotted onto a nitrocellulose membrane, with $20 \mathrm{ng}$ per lane. The membrane was stained with Ponceau S solution and blocked overnight with $5 \%$ nonfat dry milk in PBS $(137 \mathrm{mM} \mathrm{NaCl}, 2.7 \mathrm{mM} \mathrm{KCl}, 10 \mathrm{mM}$ Na2HPO4, and 2 mM KH2PO4, pH 7.4, 0.25\% Tween 20). Primary antibody dilutions in PBS were as follows: polyclonal rabbit anti-GFP (Invitrogen), 1:800; polyclonal rabbit antibodies anti-AtSEC3, 1:5000; anti-AtSEC5, 1:5000; anti-AtSEC6 (Agrisera Sweden), 1:10000; anti-AtSEC8 (Agrisera Sweden), 1:8000; anti-AtSEC10, 1:10000; anti-AtSEC15a, 1:1000 and anti-AtEXO84b, 1:1000. Appropriate secondary horseradish peroxidase conjugated antibodies (Promega, Madison, WI, USA) were applied, followed by chemiluminescent ECL detection (Amersham, GE Healthcare, Chicago, IL, USA). 
Arabidopsis seedlings (1 $\mathrm{g}$ of 10-day-old seedlings) were used for the co-immunoprecipitation of proteins. Protein complexes with GFP-SYP121-GFP, EXO70B2-GFP, GFP-EXO70B2 and free-GFP as a control were isolated using the $\mu$ MACS GFP-tagged protein isolation kit (Miltenyi Biotec) according to the manufacturer's instructions. The only exception was the utilisation of Sec $6 / 8$ buffer (Hála et al., 2008), for experiments with GFP-SYP121 and free-GFP, as a wash buffer two times and the lysis buffer provided in the kit as a third more stringent wash $(150 \mathrm{mM} \mathrm{NaCl}, 1 \%$ Triton X-100, $0.5 \%$ sodium deoxycholate, $0.1 \%$ SDS, $50 \mathrm{mM}$ Tris $\mathrm{HCl}(\mathrm{pH} 8.0)$ and subsequently a second wash buffer (20 mM Tris $\mathrm{HCl}(\mathrm{pH} 7.5))$. The elution was performed using the elution buffer provided in the kit (50 mM Tris $\mathrm{HCl}$ (pH 6.8), $50 \mathrm{mM}$ DTT, 1\% SDS, 1 mM EDTA, $0.005 \%$ bromophenol blue, $10 \%$ glycerol). To analyse bound proteins in an eluted fraction, proteins were separated using a $10 \%$ mini SDS-PAGE gel at $160 \mathrm{~V}$ for $20 \mathrm{~min}$. The samples intended for immunodetection were processed for western blotting and immunochemical detection. The samples intended for HPLC analysis were prepared as follows: the SDS-PAGE gel was stained with colloidal Coomassie Brilliant Blue (Imperial Protein Stain, Thermo Scientific), and the whole lane was cut into four parts and submitted to in-gel digestion. The gel bands were cut into 1-mm fragments that were distained using a mixture of acetonitrile and $0.1 \mathrm{M}$ ammonium bicarbonate in a 1:1 (v/v) ratio. The distained gel fragments were dried with acetonitrile, and a dithiothreitol (DTT) solution was added to reduce disulphide bonds (at $56^{\circ} \mathrm{C}$ for $45 \mathrm{~min}$ ). After removal of the DTT solution, the cubes were cooled down, and an iodoacetamide solution was added. Alkylation was performed at room temperature for $30 \mathrm{~min}$ in the dark. The gel fragments were then washed and finally dried in acetonitrile. A solution of trypsin (12.5 $\mathrm{ng} / \mu \mathrm{l}$ ) was added to the dried gel fragments and incubated at $4^{\circ} \mathrm{C}$ for $25 \mathrm{~min}$. The excess trypsin solution was removed, and a fresh solution of $50 \mathrm{mM}$ ammonium bicarbonate was added. Proteins were digested at $37^{\circ} \mathrm{C}$ for $4 \mathrm{~h}$. The solution containing the peptides was then transferred to a clean tube, and sufficient $0.1 \%$ TFA was added to cover the gel fragments and stop digestion. The gel fragments were sonicated for $15 \mathrm{~min}$ to release any peptides still present inside the gel. After sonication, both aliquots were combined, cleaned with ZipTip C18 pipette tips, and left to evaporate.

\section{Mass spectrometry}

Mass spectrometry measurements were conducted using a UHPLC Dionex Ultimate3000 RSLC nano (Dionex, Germany) connected to a mass spectrometer ESI-Q-TOF Maxis Impact (Bruker, Germany). Samples were dissolved in $8 \mu \mathrm{l}$ of water/acetonitrile/formic acid (97:3:0.1\%), and then 5 $\mu \mathrm{l}$ of this mixture was loaded onto a trap column Acclaim PepMap100 C18 (100 $\mu \mathrm{m}$ x $2 \mathrm{~cm}, 5 \mu \mathrm{m}$ particle size; Dionex, Germany) with a mobile phase A ( $0.1 \%$ formic acid in water) and a flow rate of $5 \mu \mathrm{l} / \mathrm{min}$ for $5 \mathrm{~min}$. The peptides were eluted from the trap column onto the analytical column Acclaim PepMap RSLC C18 $(75 \mu \mathrm{m} \times 150 \mathrm{~mm}, 2 \mu \mathrm{m}$ particle size $)$ by mobile phase B $(0.1 \%$ formic acid in acetonitrile) using the following gradient: 0-5 min $3 \% \mathrm{~B}, 5-35 \min 5-35 \% \mathrm{~B}, 37 \min 90 \% \mathrm{~B}$, $37-5090 \% \mathrm{~B}, 51 \mathrm{~min} 3 \% \mathrm{~B}, 51-60 \mathrm{~min} 3 \% \mathrm{~B}$. The flow rate during the gradient separation was set to $0.3 \mu \mathrm{L} / \mathrm{min}$. Peptides were eluted directly to the ESI source - Captive spray (Bruker Daltonics, Germany). All data were recorded in positive ion mode. The capillary voltage was set to $1500 \mathrm{~V}$, and the dry gas temperature was $150^{\circ} \mathrm{C}$, at a flow of $3 \mathrm{l} / \mathrm{min}$. Peptides for fragmentation were selected in the range from $400-1400 \mathrm{~m} / \mathrm{z}$. Up to five precursors could be selected from one MS spectrum; dynamic exclusion was set $0.5 \mathrm{~min}$. MS/MS spectra were recorded in the range from $50-2200 \mathrm{~m} / \mathrm{z}$. Peak lists were extracted from the raw data with Data Analysis version 4.1 (Bruker Daltonics, Germany), and proteins were identified using the Mascot server (version 2.4.1, Matrix Science). Mass spectrometry data were matched against the Arabidopsis thaliana database (downloaded from the UniProt website on 11 October 2016, 33705 sequences) complemented with common laboratory contaminants. The following parameters were set during the search: enzyme trypsin, allowance of one missed cleavage, tolerance of $10 \mathrm{ppm}$ in MS mode, and 0.05 Da in MSMS mode. The cysteine carbamidomethylation was set as a fixed modification and methionine oxidation as a variable 
modification; a Mascot decoy search was used to control the false discovery rate, which was set to $1 \%$.

Results

\section{EXO70B2 is specifically upregulated in early hours after fungal attack and accumulates at attack/papillae sites}

The EXO70 isoform EXO70B2 mRNA is upregulated after various elicitor treatments in Arabidopsis, and the protein is involved in the biogenesis of papillae (Pečenková et al., 2011; Stegmann et al., 2012). We hypothesized that the EXO70B2 isoform might be specifically employed in the anti-fungal non-host defence (Žárskỳ et al., 2013). To test our hypothesis, we compared the protein expression profile of EXO70B2 with its closest paralogue EXO70B1, the developmentally most abundant isoform EXO70A1 and endogenous constitutively expressed core subunit SEC8 after $\mathrm{Bg}$ inoculation. For this purpose, we prepared several stable lines expressing EXO70B2-GFP under the natural promoter in the exo70B2 mutant background, which complemented its compromised phenotype in penetration resistance (Fig. S1), as well as lines with natural promoter driven GFPEXO70B1 [50] expression in the exo70B1 plants complementing the exo70B1 phenotype.

The level of EXO70B2 protein increased significantly at 4 hours post-inoculation (hpi; Fig. 1) and remained unchanged even at $24 \mathrm{hpi}$ (Fig. S2). The level of EXO70A1 slightly decreased, while the level of SEC8 remained unchanged, indicative of EXO70 isoforms exchange (Fig. 1A). The GFPEXO70B1 also responded to Bg infection by upregulation, however much less than EXO70B2-GFP. Indeed, to detect the level of GFP-EXO70B1 expression in the exo70B1 plants, we had to triple the amount of loaded proteins (Fig. 1B) in comparison to EXO70B2-GFP (Fig. 1A). To exclude the influence of the positional effect of the GFP tag, we also analysed an N-terminal fusion GFPEXO70B2 variant, which responded to the fungus presence similarly to EXO70B2-GFP (Fig. S2).

To detect the earliest point of EXO70B2-GFP recruitment to the $\mathrm{Bg} / \mathrm{Arabidopsis}$ contact sites, we acquired images of infected Arabidopsis leaves every two hours, starting from the 0 point of inoculation until $24 \mathrm{hpi}$. We spotted the very first signal accumulation of EXO70B2-GFP at the fungal attack sites between 8 and 9 hpi (Fig. 1C). This time corresponds to the early deposition of papillae material (Assaad et al., 2004), and therefore, we propose that EXO70B2 is involved in the initial definition of the membrane domain for papilla biogenesis. In agreement with western blot analysis of protein levels (Fig. 1A), neither GFP-EXO70B1 nor GFP-EXO70A1 showed a similar early accumulation at the contact sites (Fig. 1C).

\section{The exocyst co-localises with the growing structure of the haustorial encasement}

Despite defensive papillae-formation, non-adapted fungi such as Bg and Ep are occasionally able to successfully penetrate a non-host plant cell and develop haustoria, which later become encased, therefore we decided to analyse the presence of the exocyst subunits in both papillae structures and haustorial encasements. Using optical scanning microscopy, we inspected the surroundings of the surface of a Bg haustorium using EXO70B2-GFP and as a representative of a core exocyst subunit SEC6-GFP expressing Arabidopsis lines 48 hpi with Bg. Both tagged subunits were found to label the area of papillae (Fig. 2A, B), but also the encasements formed around Bg haustoria (Fig. 2 C, D).

The EXO70B2-GFP signal outlined the papillary body (Fig. 2A), the encasement (Fig. 2C), but was excluded from the part of haustoria lacking the encasement, i.e., it was not present at the extrahaustorial membrane (Fig. 2C, pink arrowheads). SEC6-GFP displayed a generally more intense signal than EXO70B2-GFP, in case of both papillae and haustoria (Fig. 2B and D). The exocyst subunit localisation in papillae and haustorial encasements was further verified by lambda scanning and subsequent linear unmixing analysis (Fig. S3). These observations suggest that the EXO70B2containing exocyst complex participates in both papilla and encasement biogenesis. We also inspected the dynamics of the EXO70B2-GFP and SEC6-GFP signals within the papillae 24 hpi with $\mathrm{Bg}$. The kymographs generated from $30 \mathrm{~s}$ of 2-min-long time series showed that the localisation of 
both EXO70B2-GFP and SEC6-GFP exocyst subunits within a papilla is stationary (Fig. S4), similarly to previously observed localisation of Qa-SNARE SYP121 (Nielsen et al., 2012).

\section{Exocyst subunit mutants display abnormal papillae and impaired penetration resistance}

To further investigate exocyst role in non-host resistance, we decided to analyse the defence reaction of LOF exocyst mutant plants. We employed mutants that lack strong developmental defects, such as the knockout line (KO) sec5a-1 (GABI_731C01, Fig. S5) and the truncated mutant line sec8-m4 (Cole et al., 2005). We compared their performance with the exo70B2 KO line and as the control, we used Col-0 ecotype. By staining with trypan blue and aniline blue, we detected, along with the previously characterised and reported types of fungal propagation (regular papilla, encased haustorium and unencased haustorium; (Takemoto et al., 2006)) additional papilla structures with a vesicular halo as described previously (Pečenková et al., 2011), as well as enlarged papillae with a diameter more than twice that of a regular papilla (Fig. 3, S6).

In these abnormal papillae, we identified two distinct patterns of callose deposition. The vesicular clump category exhibited a reduction of callose and the stacking of faint callose spots around the appressorium (Fig. 3A, S7). In contrast, the enlarged papillae showed an over-accumulation of callose, as well as a larger papilla body observed under the bright field (Fig. 3A, S7). We quantified the occurrence of these papillae and penetration efficiency at 24 and $48 \mathrm{hpi}$ with $\mathrm{Bg}$ and $116 \mathrm{hpi}$ with Ep in the selected mutant lines (due to the slower spore germination of Ep, we prolonged the inoculation time for evaluation of its penetration; Fig. 3B). High penetration efficiency was correlated with cells either developing haustoria or undergoing cell death (Fig. 3A). In accordance with published data (Pečenková et al., 2011), exo70B2 plants demonstrated a higher occurrence of deviated papillae, as well as an increased penetration efficiency of Ep (Fig. 3B). The sec5a-1 and sec8-m4 mutant lines showed significant increases in both the deviated papillae and the penetration rate (Fig. 3B).

\section{Time course comparison of callose deposition and haustorium formation between exocyst and syp121 mutants}

The observed morphological changes of papillae indicate EXO70B2-containing exocyst complex (including SEC8) is involved in papillae biogenesis and callose deposition within papillae. In order to quantify a range of the exocyst disruption effect on the callose deposition, we quantified the Bginduces callose punctae on leaves of mutants exo70B2 and sec8-m4 in comparison to the wild type control, and also LOF syp121, a mutant of the SNARE protein that has been shown to be important for timely deposition of callose into papillae (Collins et al., 2003; Assaad et al., 2004) (Fig. 4A). In addition, we followed the time course development of haustoria in living cells in these lines (Fig. 4B).

In our experiment, the sec8- $m 4$ mutant displayed faster penetration by Bg than the WT, but still slower penetration than the syp121 mutant (manifested as formed haustoria number; Fig. 4B). The higher penetration success of $\mathrm{Bg}$ in syp 121 has been associated with the delay in callose deposition in early papilla development. We observed a comparable delay in callose deposition for all three sec8$m 4$, exo $70 B 2$ and syp 121 single LOF mutants at 10 hpi. Surprisingly, the exo $70 B 2$ mutant exhibited the strongest decrease in callose deposition, which remained apparent until the later time points after inoculation.

\section{EXO70B2 can interact with SYP121}

Results of the analysis of callose deposition indicate a functional overlap and a connection between the EXO70B2/exocyst- and SYP121-driven secretory pathways, similarly to the situation in yeast, and also in seedlings of $A$. thaliana (Sivaram et al., 2005; Larson et al., 2020). To examine the possibility that the two complexes might interact in the non-host anti-fungal defence via EXO70B2SYP121 direct interaction, we employed a yeast-two hybrid system. We used the truncated construct 
SYP121 $\Delta \mathrm{C}$ (free of the transmembrane domain) and besides EXO70B2 also several other exocyst complex subunits (AD-SYP121 $\triangle$ C/BD-EXO70A1, EXO70B1, EXO70B2, EXO70H1, SEC3a, SEC5a, SEC6, SEC8, SEC10a, SEC15b, EXO84b, and the pair SEC3a/EXO70A1 was used as a positive control; Fig. 5A). We detected only a weak interaction of SYP121 $\triangle \mathrm{C}$ with EXO70B2 and EXO70B1 (A). To verify the interaction in planta, we examined the GFP-SYP121 bound fraction with available anti-exocyst antibodies. In the eluate, we detected the presence of two exocyst core subunits, SEC6 and SEC3 (Fig. 5B), confirming the ability of the exocyst complex to associate with SYP121 in Arabidopsis. However, using the opposite approach, where EXO70B2-GFP after plant treatment with $\mathrm{Bg}$ was used as bait for co-immunoprecipitation followed by sensitive HPLC MS/MS analysis, did not reveal the presence of SYP121 (Supplementary Table 2).

\section{EXO70B2 and SYP121 cooperate in papillae biogenesis}

Since EXO70B2 and SYP121 both regulate early papillae biogenesis and are capable of direct interaction, we searched for evidence of their functional cooperation. Using the stable transgenic Arabidopsis lines, we examined the co-localisation of mRuby2-EXO70B2 with GFP-SYP121 16 hpi with $\mathrm{Bg}$ (Fig. 6A). Both proteins co-localised at the papilla periphery (white colour), and their fragile co-localisation disappeared after plasmolysis (Fig. S8). Unlike GEP-SYP121, mRuby2-EXO70B2 was absent from the apoplastic space of papillae (Fig. S8).

We then inspected the localisation patterns of EXO70B2 and SYP121 in counterpart mutant lines backgrounds. First, we followed the localisation of GFP-SYP121 in papillae at 24 hpi with Bg in WT and exo70B2 mutant plants (Fig. 6B). In the WT background, the maximum intensity projection of GFP-SYP121 signal appeared due to its extracellular localisation as a filled circular area of variable diameter (Fig. 6B), as described previously (Collins et al., 2003; Assaad et al., 2004). However, in the exo70B2 mutant background, GFP-SYP121 labelled a significantly smaller papilla area (Fig. 6B). We also noticed that the GFP-SYP121 signal in exo70B2 plants often showed a thin doughnut-like pattern (hollow circle) at plant/fungal contact sites, indicative of missing extracellular localisation as observed in the WT (Fig. 6B). Second, we analysed the localisation of EXO70B2-GFP in the WT and syp 121 mutant background. In this case, we did not observe a change in the pattern of the signal itself, but the area of the EXO70B2-GFP papillae was smaller in syp121 compared with WT (Fig. 6C). We decided to further test the relevance of the interaction of EXO70B2 and SYP121 to penetration resistance at the genetic level. We examined the penetration resistance of the double mutant exo70B2/syp 121 to $\mathrm{Bg}$ in comparison to the single syp121 and exo70B2 mutants (the appearance of the infected plants is shown in Fig. S9). The exo70B2/syp121 double mutant had a significantly decreased number of regular papillae in comparison to the single syp121 (Fig. S9). Despite an absence of a significant difference in the total number of haustoria in living cells (Fig. S9), the exo70B2/syp121 double mutant had a decreased number of encased haustoria and an increased number of unencased haustoria compared with the syp121 single mutant (Fig. 6D). In our set of tested mutants, we observed that both single and double mutants showed similar delays in callose deposition at earlier time points p.i.; however, at later time points the additional loss of EXO70B2 in syp121 slightly but steadily dampened the otherwise increased level of callose punctae (Fig. 6E).

\section{Discussion}

\section{Exocyst is present in membrane domain of papillae and encasements around haustoria}

The exocyst roles in regulation of plant cell secretion and polarity, and also surprising involvement in autophagy, imply its participation also in defence responses (Žárskỳ et al., 2013). Indeed, in accord with the published observations of EXO70B2, the isoform of EXO70 subunit of the exocyst complex, mRNA upregulation by PAMPs (Pečenková et al., 2011; Stegmann et al., 2012), we documented early protein upregulation and focal accumulation of EXO70B2-GFP and core exocyst subunit SEC6GFP in areas of Blumeria penetration attempts and papilla and haustorium encasement formation (Fig. 1, 2). We showed that this enhanced localisation was a specific regulated response since the 
increase in the EXO70B2-GFP protein level correlated with mild EXO70A1 depletion, while the level of another core subunit SEC8 remained unchanged (Fig. 1A). Our data also support the specific role of EXO70B2 in the biogenesis of papillae over its closest paralogue, the EXO70B1, while, as has been shown recently, both cooperate in FLS2 signalling (Wang et al., 2020). Clearly, the localisation and expression pattern shows differences from the rest of the complex, which, along with the previous results documenting its ability to interact with exocyst core, suggests that EXO70B2 employ only proportionate part of the core subunits. Interestingly, for the analysed exocyst subunits, the localisation in the membrane domain of papillae was found to be persistent over time (Fig. S4). A similarly stable localisation of exocyst subunits has been observed during secondary cell wall deposition in tracheary elements or trichomes (Kulich et al., 2015, 2018; Vukašinović et al., 2017), in contrast to the dynamic exocyst localisation at the lateral PM domains of rhizodermal cells (Fendrych et al., 2013). These data suggest that exocyst tends to stay trapped in secretory domains involved in deposition of cell-wall components, particularly callose which is a component of both papilla and encasement around a haustorium.

\section{Impaired balance of the exocyst complex compromises penetration resistance}

A disruption of the plant exocyst complex leads to severe growth phenotypes (Synek et al., 2006; Hála et al., 2008; Kulich et al., 2013; Žárskỳ et al., 2013). To study the specific role of the exocyst in anti-fungal defence, but to avoid pleiotropic mutation effects of full exocyst LOF, we worked, along with the immunity-related mutant exo70B2, also with weak allele lines of Arabidopsis core exocyst subunits sec8-m4 and sec5a-1. Using these mutants, we were able to describe new Arabidopsis-Bg interaction phenotypic deviation, apart from the previously described fungal stages found for WT (Takemoto et al., 2006). In addition to the previously characterised vesiculated papillae (Pečenková et al., 2011), we newly described the presence of enlarged papillae. These abnormal papillae in the exocyst mutants manifested uneven deposition of callose consisting of either faint callose signals or excessive callose deposition on the contrary (Fig. 3). Such enlarged papillae with excess callose resembled the phenotype of the Arabidopsis line overexpressing PMR4 callose synthase (Blümke et al., 2013). In the core subunit sec8- $m 4$ mutant, we observed stronger penetration defects than in the exo70B2 mutant. This finding may reflect a general role of SEC8 in the maintenance of the secretory efficiency, in contrast to the specific requirement for EXO70B2 in defence. Intriguingly, even though the exo70B2 and sec $8-m 4$ mutants, similarly to the syp121 mutant, exhibited a delay in early callose deposition, their penetration resistance was not compromised as drastically as in the case of syp121 (Fig. 4). We could speculate that these milder phenotypes in penetration resistance were the result of a compensation by another EXO70 isoform, as well as the partial function of the truncated SEC8 in the case of sec8-m4. On the other hand, there are multiple reports on Arabidopsis mutants defective in callose deposition whose overall resistance is not compromised (Aist, 1976; Jacobs et al., 2003; Nishimura et al., 2003), therefore our data might also support a view that the deposition of callose may not be of key importance for the final defence outcome. This assumption is supported by another observation, wherein the double mutant exo70B2/syp121 was the deposition of stress-related callose dampened even more than in syp121 single mutant, however without a major change in total numbers of penetrated cells (Fig. 6, S9).

It has been previously suggested that the callose is loaded into papillae via multivesicular bodies rather than being synthesized within the cell wall apposition (Böhlenius et al., 2010). This phenomenon would imply an involvement of the exocyst in non-canonical secretion to the papillae extracellular space that would involve callose but also GFP-SYP121 as a non-functional cargo (Nielsen et al., 2012), and very probably other defence-related cell wall material and phytoalexins. This theory is supported by the association of GFP tagged EXO70B2 with MVB-related RabG3C in pathogen-treated plants (Supplementary Table S2), however this needs to be verified in future, as well as if and how the EXO70B2 papillae function is related to phosphorylation status and interaction 
with ATG8 (Brillada et al., 2021). In agreement with these observations is the employment of EXO70B2 in trafficking seemingly other than canonical exocytosis.

\section{The exocyst complex affects SYP121 SNARE in antifungal defence}

In this report, we showed that SYP121 and EXO70B2 are capable of direct interaction, they colocalise, affect each other's localisation and have additive defence effects in functional genetic analyses. The cooperation of the two proteins could also be extrapolated based on their co-expression and interactions with SNAP33 (Pečenková et al., 2011; Bozkurt et al., 2015). Interestingly, our y2h test revealed that the soluble part of SYP121 was able to interact with EXO70B1 but not EXO70A1 (Fig. 5A). Although we did not find a relevant role for EXO70B1 or for EXO70A1 in non-host resistance, we admit that other EXO70 isoforms might cooperate with SYP121 in defence. Our EXO70B2/SYP121 cooperation model, despite several positive tests, was not supported by HPLCMS/MS analysis of proteins bound to EXO70B2-GFP. A reason for this is most probably a transient nature of the exocyst (peripheral membrane complex)-SNARE (integral membrane proteins) interaction. Regarding the relationship of EXO70B2 and SYP121, the most relevant distinction between the two proteins lies within the observation that unlike GFP-SYP121, EXO70B2-GFP is not secreted into the extracellular papillar space while it does contribute to GFP-SYP121 unconventional extracellular papillar localisation, the relevance of which remains to be analysed. Based on the interaction capability, mutual dependent localization as well as additive effect of the exo70B2/syp 121 double mutant, we conclude that EXO70B2 and SYP121 cooperate in the secretory pathway that drives cell wall and defence material towards the papillae and encasements in response to the fungal attack (Fig. 7).

Our data show that the exocyst, mainly its EXO70B2-containing variant, plays a key role in papilla and haustorial encasement build-up, including callose secretion, during non-host defence. The exclusive role of the EXO70B2 subunit in papillae and encasement formation is probably executed via a functional relationship with the SYP121-carrying SNARE complex. However, in addition to the usual consideration of the existence of alternative redundant complex variants, our data do not exclude the existence of diversified exocyst and SNARE pathways involved in defence. The EXO70B2-dependent SYP121 localisation to the paramural space vs. the absence of the exocyst in it raises especially interesting questions concerning the mechanistic details and time sequence of the action of the exocyst vs. SNARE, which requires further research.

\section{Supplementary data}

Supplementary Fig. 1. The EXO70B2-GFP and GFP-EXO70B2 complementation of the exo70B2 mutant phenotype.

Supplementary Fig. 2. Expression level of exocyst subunits after Bg inoculation.

Supplementary Fig. 3. Linear unmixing analysis of GFP-tagged exocyst subunits in papillae and haustoria.

Supplementary Fig. 4. Exocyst dynamics in papillary membrane domains.

Supplementary Fig. 5. Verification of the KO sec5a-1 mutant.

Supplementary Fig. 6. Detailed visualisation of $\mathrm{Bg} /$ Arabidopsis interactions using trypan blue staining.

Supplementary Fig. 7. Detailed visualisation of Bg/Arabidopsis interactions using aniline blue staining.

Supplementary Fig. 8. EXO70B2 does not follow SYP121 into apoplast in defensive papilla. 
Supplementary Fig. 9. Analysis of exo70B2/syp121 double mutant reaction to Bg infection.

Supplementary Table 1. List of primers and constructs used in the study.

Supplementary Table 2. Identified proteins in EXO70B2-GFP bound fraction.

Acknowledgements

This work was supported by Czech Science Foundation (CSF/GACR) project 19-02242J and terminated GA16-34887L, a terminated student project GAUK-1102214 (Charles University Grant Agency), and the Fund-Project "Centre for Experimental Plant Biology": No. CZ.02.1.01/0.0/0.0/16_019/0000738 of MEYS CR. The Institute of Experimental Botany (IEB) Imaging Facility is supported by the Operational Programme Prague Competitiveness (OPPC) CZ.2.16/3.1.00/21519 and Czech-BioImaging large RI project LM2015062 funded by MEYS CR.

\section{Author contributions}

Conceived and designed the experiments: JO, TP, VZ. Performed the experiments: JO, TP. Analysed the data: JO. Contributed reagents/materials/analysis tools: JO, TP, JS, JŠ, IK, PD. Wrote the paper: JO, TP, JS, VZ

\section{Data availability statement}

All data supporting the findings of this study are available within the paper and within its supplementary materials published online.

The data supporting the findings of this study are available from the corresponding author (Tamara Pečenková) upon request. 


\section{References}

Aist JR. 1976. Papillae and related wound plugs of plant cells. Annual Review of Phytopathology 14, 145-163.

An Q, van Bel AJ, Hückelhoven R. 2007. Do plant cells secrete exosomes derived from multivesicular bodies? Plant signaling \& behavior 2, 4-7.

Assaad FF, Qiu J-L, Youngs H, Ehrhardt D, Zimmerli L, Kalde M, Wanner G, Peck SC, Edwards H, Ramonell K. 2004. The PEN1 syntaxin defines a novel cellular compartment upon fungal attack and is required for the timely assembly of papillae. Molecular biology of the cell 15, $5118-5129$.

Bestwick CS, Bennett MH, Mansfield JW. 1995. Hrp mutant of Pseudomonas syringae pv phaseolicola induces cell wall alterations but not membrane damage leading to the hypersensitive reaction in lettuce. Plant Physiology 108, 503-516.

Blümke A, Somerville SC, Voigt CA. 2013. Transient expression of the Arabidopsis thaliana callose synthase PMR4 increases penetration resistance to powdery mildew in barley. Advances in Bioscience and Biotechnology 2013.

Böhlenius H, Mørch SM, Godfrey D, Nielsen ME, Thordal-Christensen H. 2010. The multivesicular body-localized GTPase ARFA1b/1c is important for callose deposition and ROR2 syntaxin-dependent preinvasive basal defense in barley. The Plant Cell 22, 3831-3844.

Bozkurt TO, Belhaj K, Dagdas YF, Chaparro-Garcia A, Wu C-H, Cano LM, Kamoun S. 2015. Rerouting of plant late endocytic trafficking toward a pathogen interface. Traffic 16, 204 226.

Brillada C, Teh O-K, Ditengou FA, et al. 2021. Exocyst subunit Exo70B2 is linked to immune signaling and autophagy. The Plant Cell 33, 404-419.

Chowdhury J, Henderson M, Schweizer P, Burton RA, Fincher GB, Little A. 2014.

Differential accumulation of callose, arabinoxylan and cellulose in nonpenetrated versus penetrated papillae on leaves of barley infected with Blumeria graminis f. sp. hordei. New Phytologist 204, $650-660$.

Clough SJ, Bent AF. 1998. Floral dip: a simplified method for Agrobacterium-mediated transformation of Arabidopsis thaliana. The plant journal 16, 735-743.

Cole RA, Synek L, Zarsky V, Fowler JE. 2005. SEC8, a subunit of the putative Arabidopsis exocyst complex, facilitates pollen germination and competitive pollen tube growth. Plant physiology 138.

Collins NC, Thordal-Christensen H, Lipka V, Bau S, Kombrink E, Qiu J-L, Hückelhoven R, Stein M, Freialdenhoven A, Somerville SC. 2003. SNARE-protein-mediated disease resistance at the plant cell wall. Nature 425, 973-977.

Consonni C, Humphry ME, Hartmann HA, Livaja M, Durner J, Westphal L, Vogel J, Lipka V, Kemmerling B, Schulze-Lefert P. 2006. Conserved requirement for a plant host cell protein in powdery mildew pathogenesis. Nature genetics 38, 716-720.

Cvrcková F, Grunt M, Bezvoda R, Hála M, Kulich I, Rawat A, Zarsky V. 2012. Evolution of the land plant exocyst complexes. Frontiers in plant science 3, 159. 
Drdova EJ, Synek L, Pečenková T, Hála M, Kulich I, Fowler JE, Murphy AS, Žárskỳ V. 2013. The exocyst complex contributes to PIN auxin efflux carrier recycling and polar auxin transport in Arabidopsis. The Plant Journal 73, 709-719.

Dubuke ML, Maniatis S, Shaffer SA, Munson M. 2015. The exocyst subunit Sec6 interacts with assembled exocytic SNARE complexes. Journal of Biological Chemistry 290, 28245-28256.

Ebine K, Fujimoto M, Okatani Y, Nishiyama T, Goh T, Ito E, Dainobu T, Nishitani A, Uemura T, Sato MH. 2011. A membrane trafficking pathway regulated by the plant-specific RAB GTPase ARA6. Nature cell biology 13, 853-859.

Elias M, Drdova E, Ziak D, Bavlnka B, Hala M, Cvrckova F, Soukupova H, Zarsky V. 2003. The exocyst complex in plants. Cell biology international 27, 199-201.

Eschrich W, Currier HB. 1964. Identification of cauose by its diachrome and fluorochrome reactions. Stain Technology 39, 303-307.

Fendrych M, Synek L, Pečenková T, Drdová EJ, Sekereš J, Rycke R de, Nowack MK, Žárskỳ V. 2013. Visualization of the exocyst complex dynamics at the plasma membrane of Arabidopsis thaliana. Molecular biology of the cell 24, 510-520.

Fendrych M, Synek L, Pečenková T, Toupalová H, Cole R, Drdová E, Nebesářová J, Šedinová M, Hála M, Fowler JE. 2010. The Arabidopsis exocyst complex is involved in cytokinesis and cell plate maturation. The Plant Cell 22, 3053-3065.

Guo W, Roth D, Gatti E, De Camilli P, Novick P. 1997. Identification and characterization of homologues of the Exocyst component Sec10p. FEBS letters 404, 135-139.

Hála M, Cole R, Synek L, Drdová E, Pečenková T, Nordheim A, Lamkemeyer T, Madlung J, Hochholdinger F, Fowler JE. 2008. An exocyst complex functions in plant cell growth in Arabidopsis and tobacco. The Plant Cell 20, 1330-1345.

Heard W, Sklenář J, Tome DF, Robatzek S, Jones AM. 2015. Identification of regulatory and cargo proteins of endosomal and secretory pathways in Arabidopsis thaliana by proteomic dissection. Molecular \& Cellular Proteomics 14, 1796-1813.

Heath MC, Heath IB. 1971. Ultrastructure of an immune and a susceptible reaction of cowpea leaves to rust infection. Physiological Plant Pathology 1, 277-287.

Hsu S-C, Ting AE, Hazuka CD, Davanger S, Kenny JW, Kee Y, Scheller RH. 1996. The mammalian brain rsec6/8 complex. Neuron 17, 1209-1219.

Ishiga Y, Ishiga T, Uppalapati SR, Mysore KS. 2011. Arabidopsis seedling flood-inoculation technique: a rapid and reliable assay for studying plant-bacterial interactions. Plant methods 7, 32.

Jacobs AK, Lipka V, Burton RA, Panstruga R, Strizhov N, Schulze-Lefert P, Fincher GB. 2003. An Arabidopsis callose synthase, GSL5, is required for wound and papillary callose formation. The Plant Cell 15, 2503-2513.

Jones JD, Dangl JL. 2006. The plant immune system. nature 444, 323-329.

Kato N, Fujikawa Y, Fuselier T, Adamou-Dodo R, Nishitani A, Sato MH. 2010. Luminescence detection of SNARE-SNARE interaction in Arabidopsis protoplasts. Plant molecular biology 72, 433-444. 
Kubátová Z, Pejchar P, Potockỳ M, Sekereš J, Žárskỳ V, Kulich I. 2019. Arabidopsis trichome contains two plasma membrane domains with different lipid compositions which attract distinct EXO70 subunits. International journal of molecular sciences 20, 3803.

Kulich I, Cole R, Drdová E, Cvrčková F, Soukup A, Fowler J, Žárskỳ V. 2010. Arabidopsis exocyst subunits SEC8 and EXO70A1 and exocyst interactor ROH1 are involved in the localized deposition of seed coat pectin. New Phytologist 188, 615-625.

Kulich I, Pečenková T, Sekereš J, Smetana O, Fendrych M, Foissner I, Höftberger M, Žárskỳ V. 2013. Arabidopsis exocyst subcomplex containing subunit EXO70B1 is involved in autophagyrelated transport to the vacuole. Traffic 14, 1155-1165.

Kulich I, Vojtíková Z, Glanc M, Ortmannová J, Rasmann S, Žárskỳ V. 2015. Cell wall maturation of Arabidopsis trichomes is dependent on exocyst subunit EXO70H4 and involves callose deposition. Plant Physiology 168, 120-131.

Kulich I, Vojtíková Z, Sabol P, Ortmannová J, Neděla V, Tihlaříková E, Žárskỳ V. 2018. Exocyst subunit EXO70H4 has a specific role in callose synthase secretion and silica accumulation. Plant physiology 176, 2040-2051.

Kwon C, Neu C, Pajonk S, Yun HS, Lipka U, Humphry M, Bau S, Straus M, Kwaaitaal M, Rampelt H. 2008. Co-option of a default secretory pathway for plant immune responses. Nature 451, 835-840.

Larson ER, Ortmannová J, Donald NA, Alvim J, Blatt MR, Žárský V. 2020. Synergy among Exocyst and SNARE Interactions Identifies a Functional Hierarchy in Secretion during Vegetative Growth. The Plant Cell 32, 2951-2963.

Lee H-A, Lee H-Y, Seo E, Lee J, Kim S-B, Oh S, Choi E, Choi E, Lee SE, Choi D. 2017.

Current understandings of plant nonhost resistance. Molecular Plant-Microbe Interactions 30, 5-15.

Lipka V, Dittgen J, Bednarek P, Bhat R, Wiermer M, Stein M, Landtag J, Brandt W, Rosahl S, Scheel D. 2005. Pre-and postinvasion defenses both contribute to nonhost resistance in Arabidopsis. science 310, 1180-1183.

Lipka U, Fuchs R, Lipka V. 2008. Arabidopsis non-host resistance to powdery mildews. Current opinion in plant biology 11, 404-411.

Liu D, Li X, Shen D, Novick P. 2018. Two subunits of the exocyst, Sec3p and Exo70p, can function exclusively on the plasma membrane. Molecular biology of the cell 29, 736-750.

Meyer D, Pajonk S, Micali C, O'Connell R, Schulze-Lefert P. 2009. Extracellular transport and integration of plant secretory proteins into pathogen-induced cell wall compartments. The Plant Journal 57, 986-999.

Micali CO, Neumann U, Grunewald D, Panstruga R, O'Connell R. 2011. Biogenesis of a specialized plant-fungal interface during host cell internalization of Golovinomyces orontii haustoria. Cellular microbiology 13, 210-226.

Nielsen ME, Feechan A, Böhlenius H, Ueda T, Thordal-Christensen H. 2012. Arabidopsis ARF-GTP exchange factor, GNOM, mediates transport required for innate immunity and focal accumulation of syntaxin PEN1. Proceedings of the National Academy of Sciences 109, 1144311448 .

Nielsen ME, Jürgens G, Thordal-Christensen H. 2017. VPS9a activates the Rab5 GTPase ARA7 to confer distinct pre-and postinvasive plant innate immunity. The Plant Cell 29, 1927-1937. 
Nielsen ME, Thordal-Christensen H. 2012. Recycling of Arabidopsis plasma membrane PEN1 syntaxin. Plant signaling \& behavior 7, 1541-1543.

Nielsen ME, Thordal-Christensen H. 2013. Transcytosis shuts the door for an unwanted guest. Trends in plant science 18, 611-616.

Nishimura MT, Stein M, Hou B-H, Vogel JP, Edwards H, Somerville SC. 2003. Loss of a callose synthase results in salicylic acid-dependent disease resistance. Science 301, 969-972.

Pečenková T, Hála M, Kulich I, Kocourková D, Drdová E, Fendrych M, Toupalová H, Žárskỳ V. 2011. The role for the exocyst complex subunits Exo70B2 and Exo70H1 in the plant-pathogen interaction. Journal of experimental botany 62, 2107-2116.

Rutter BD, Innes RW. 2017. Extracellular vesicles isolated from the leaf apoplast carry stressresponse proteins. Plant physiology $\mathbf{1 7 3}, 728-741$.

Schmelzer E. 2002. Cell polarization, a crucial process in fungal defence. Trends in plant science 7, 411-415.

Sekereš J, Pejchar P, Šantrluuček J, Vukašinović N, Žárskỳ V, Potockỳ M. 2017. Analysis of exocyst subunit EXO70 family reveals distinct membrane polar domains in tobacco pollen tubes. Plant Physiology 173, 1659-1675.

Sivaram MV, Saporita JA, Furgason ML, Boettcher AJ, Munson M. 2005. Dimerization of the exocyst protein Sec6p and its interaction with the t-SNARE Sec9p. Biochemistry 44, 6302-6311.

Stegmann M, Anderson RG, Ichimura K, Pecenkova T, Reuter P, Žárskỳ V, McDowell JM, Shirasu K, Trujillo M. 2012. The ubiquitin ligase PUB22 targets a subunit of the exocyst complex required for PAMP-triggered responses in Arabidopsis. The Plant Cell 24, 4703-4716.

Stein M, Dittgen J, Sánchez-Rodríguez C, Hou B-H, Molina A, Schulze-Lefert P, Lipka V, Somerville S. 2006. Arabidopsis PEN3/PDR8, an ATP binding cassette transporter, contributes to nonhost resistance to inappropriate pathogens that enter by direct penetration. The Plant Cell 18, 731-746.

Synek L, Schlager N, Eliáš M, Quentin M, Hauser M-T, Žárskỳ V. 2006. AtEXO70A1, a member of a family of putative exocyst subunits specifically expanded in land plants, is important for polar growth and plant development. The Plant Journal 48, 54-72.

Synek L, Vukašinović N, Kulich I, Hála M, Aldorfová K, Fendrych M, Žárskỳ V. 2017. EXO70C2 is a key regulatory factor for optimal tip growth of pollen. Plant Physiology 174, 223240.

Takemoto D, Jones DA, Hardham AR. 2006. Re-organization of the cytoskeleton and endoplasmic reticulum in the Arabidopsis pen1-1 mutant inoculated with the non-adapted powdery mildew pathogen, Blumeria graminis f. sp. hordei. Molecular plant pathology 7, 553-563.

TerBush DR, Maurice T, Roth D, Novick P. 1996. The Exocyst is a multiprotein complex required for exocytosis in Saccharomyces cerevisiae. The EMBO journal 15, 6483-6494.

Vogel J, Somerville S. 2000. Isolation and characterization of powdery mildew-resistant Arabidopsis mutants. Proceedings of the National Academy of Sciences 97, 1897-1902.

Vukašinović N, Cvrčková F, Eliáš M, Cole R, Fowler JE, Žárskỳ V, Synek L. 2014. Dissecting a hidden gene duplication: the Arabidopsis thaliana SEC10 locus. PLoS One 9. 
Vukašinović N, Oda Y, Pejchar P, Synek L, Pečenková T, Rawat A, Sekereš J, Potockỳ M, Žárskỳ V. 2017. Microtubule-dependent targeting of the exocyst complex is necessary for xylem development in Arabidopsis. New Phytologist 213, 1052-1067.

Wang W, Liu N, Gao C, Cai H, Romeis T, Tang D. 2020. The Arabidopsis exocyst subunits EXO70B1 and EXO70B2 regulate FLS2 homeostasis at the plasma membrane. New Phytologist 227, 529-544.

Yue P, Zhang Y, Mei K, Wang S, Lesigang J, Zhu Y, Dong G, Guo W. 2017. Sec3 promotes the initial binary t-SNARE complex assembly and membrane fusion. Nature communications $\mathbf{8}, 1-12$.

Žárskỳ V, Cvrčková F, Potockỳ M, Hála M. 2009. Exocytosis and cell polarity in plants-exocyst and recycling domains. New Phytologist 183, 255-272.

Žárskỳ V, Kulich I, Fendrych M, Pečenková T. 2013. Exocyst complexes multiple functions in plant cells secretory pathways. Current opinion in plant biology 16, 726-733.

Zeyen RJ, Kruger WM, Lyngkjær MF, Carver TL. 2002. Differential effects of D-mannose and 2-deoxy-D-glucose on attempted powdery mildew fungal infection of inappropriate and appropriate G ramineae. Physiological and molecular plant pathology 61, 315-323.

Zhu Y, Wu B, Guo W. 2019. The role of Exo70 in exocytosis and beyond. Small GTPases 10, $331-335$.

\section{Figure legend}

Figure 1. EXO70B2 expression dynamics after Bg inoculation. (A) Protein levels of EXO70A1, SEC8 and EXO70B2-GFP in the complemented exo70B2 mutant at 0, 4, 8 hpi. (B) Protein level of GFP-EXO70B1 in the exo70B1 mutant at 0, 4, 8 hpi. Proteins were isolated from 4-week-old plants. Anti-GFP, anti-EXO70A1 and anti-SEC8 antibodies were used. (C) The early focal accumulation of EXO70B2-GFP signal in the cytoplasm in vesicle-like dots in comparison to the absent signal of GFP-EXO70B1 and a faint signal of GFP-EXO70A1 at $8 \mathrm{hpi}$ with Bg. All images represent a single optical section obtained by confocal spinning disc microscopy. All constructs were expressed under their natural promotors. The white dashed line indicates the fungus. The scale bar represents $10 \mu \mathrm{m}$.

Figure 2. Exocyst localisation in areas of papillae and haustorial encasements. The panels represent brightfield and signal intensity analysis of the three stages of defensive structure growth: papilla (A, B) and enclosing encasement (C, D). The EXO70B2 signal surrounds the papilla (A) and the encasement (C). The SEC6-GFP signal strongly marks the papilla (B) and the encasement (D). Plants were treated for 48 hours with $B g$. Pink arrowheads denote the haustorium encasement ends, where the exocyst subunit signal disappears. The white dashed line outlines fungal structures. The yellow arrowheads indicate penetration sites. All images represent a single optical section obtained by confocal microscopy. The scale bar represents $10 \mu \mathrm{m}$; the scale bar of signal intensities on the right.

Figure 3. Category and quantification of the interactions between exocyst mutants and nonadapted pathogens. From the top: the first row shows independent $\mathrm{Bg}$ (Ep)/Arabidopsis interactions visualized by trypan blue staining; the second row displays aniline blue staining; the third row represents a schematic model of the interaction. $\mathrm{Bg}$ and Ep evoke the same types of interactions in Arabidopsis: regular papilla; abnormal papillae / either with a vesicular halo or enlarged papilla; penetrated cells / either unencased or encased haustorium. (B) The data show the mean of the abnormal papillae and penetrated cells by tested pathogens at different time points. For each column in the graph, the area complementary to $100 \%$ represents all interactions with spores that did not 
provoke any reaction of the plant. The experiment was repeated 3 times with a similar trend. In each experiment, the $\mathrm{N}$ represents 5 quantified leaves, 100 spores were counted per leaf. Stars indicate significant differences calculated by one-way ANOVA with the post-hoc Tukey-Kramer Honestly Significant Difference (HSD) test, $* *$ Z-score $\mathrm{p}<0.01, *$ Z-score $\mathrm{p}<0.05$. For the schematic: A appressorium; GT germ tube; S spore; CW cell wall; PM plasma membrane; P papilla; EHM extrahaustorial membrane; $\mathrm{H}$ haustorium; E encasement. Yellow arrows mark outer borders of plant cell defensive structures. The dashed line outlines fungal structures. Scale bars represent $5 \mu \mathrm{m}$.

Figure 4. Effects of exocyst mutations on the occurrence of a haustorium and callose deposition in defence against Bg. (A) The frequency of callose positive punctae counted on a defined leaf area at sites of Bg attack was followed at 8, 10, 12, 24 hpi. (B) The frequency of developed haustoria in cells of WT, syp 121, exo70B2 and sec8- $m 4$ leaves. Each experiment was repeated 3 times with similar results, $\mathrm{N}$ represents 5 leaves. The asterisks indicate significant difference from WT at each time point. Statistical significance was calculated using one-way ANOVA with the post-hoc TukeyKramer HSD test, Z score $\mathrm{p}<0.05$. Error bars represent the standard error.

Figure 5. EXO70B2 subunit interacts with SYP121 in defence. (A) Direct interaction between the exocyst and SYP121 was examined in a yeast two-hybrid assay. Yeasts containing both the binding (BD) and activation domain (AD) were plated on the double selection SD-Leu, -Trp or on the SDHis, Trp, Ade, Leu plates and allowed to grow for 6 days. For each pair, negative control transformations with empty counterpart AD or BD plasmids were performed. (B) The seedlings expressing GFP-SYP121 or free GFP were used for anti-GFP co-immunoprecipitation and the bound fraction was tested for the exocyst subunits presence by immunoblotting. TE; total extract, IP immuno-precipitated proteins.

Figure 6. EXO70B2 subunit and SYP121 SNARE cooperate in papillae biogenesis. (A) Colocalisation of mRuby2-EXO70B2 and GFP-SYP121 in the membrane domain of a defensive papilla. The transgenic plants with the mRuby2-EXO70B2 and GFP-SYP121 were observed at 16 hpi with $\mathrm{Bg}$; images represent one of 15 analysed papillae with similar localisation. Pearson's correlation graph of mRuby2-EXO70B2 (Ch1) and GFP-SYP121 (Ch2) is shown on the right. (B) Representative images and quantification of GFP-SYP121 signal area in papillae in Col-0 and exo70B2 mutant backgrounds. The $\mathrm{N}$ is equal to $30 \mathrm{Z}$-stack images, consistent with $12.5 \mathrm{um}$ thickness, for each genotype. The data were processed via two-tail T-test on $\mathrm{p}<0.01$ value. (C) Complementary to panel $\mathrm{B}$, the image and quantification of EXO70B2-GFP signal area in Col-0 and syp121. Analysed with the same parameters as in B. The white dashed line marks the cross-section used for analysis. All images represent single plane sections. The scale bars represent $5 \mu \mathrm{m}$. (D-E) Analysis of Col-0, syp 121, exo70B2 and exo70B2/syp 121 mutant reactions to Bg infection. Quantification of encased and unencased haustoria present in living cells, $\mathrm{N}$ is equal to 5 leaves, 100 spores were counted per leaf. Small letters indicate statistical differences analysed by ANOVA post-hoc Tukey-Kramer (HSD) $\mathrm{Z}$ score $\mathrm{p}<0.01$. Error bars represent standard deviations. (E) Accumulation of callose punctae beneath fungal contact sites per square $\mathrm{cm}$ of leaf. The $\mathrm{N}$ is equal to 5-8 leaves. The error bars represent standard deviations.

Figure 7. Schematic of exocyst/SYP121 cooperation in defence against Bg. The schematic on the left shows a germinated spore and host cell papilla formation; the schematic on the right shows a spore forming haustoria after successful penetration into the host cell; the host cell responds by encasement formation. The cell uses at least three different secretory pathways with respect to EXO70B2 and SYP121 involvement: EXO70B2/SYP121-dependent, EXO70X/SYP121-dependent and EXO70B2-dependent and SYP121-independent. These pathways may also include the recycling and transcytosis of vesicles. We suggest that the presence of a particular EXO70 in the complex helps the exocyst to distinguish the membrane domain of defensive structures from other PM domains. The EXO70B2-containing exocyst may help to establish the papilla membrane domain for SYP121, and 
similarly in encasement formation also for another SNARE participant. A appressorium, CW cell wall, E encasement, EHM extrahaustorial membrane, ER endoplasmic reticulum, G/TGN Golgi/trans Golgi network, $\mathrm{H}$ haustorium, $\mathrm{N}$ nucleus, $\mathrm{P}$ papilla, $\mathrm{PM}$ plasma membrane, S spore, V vacuole. 


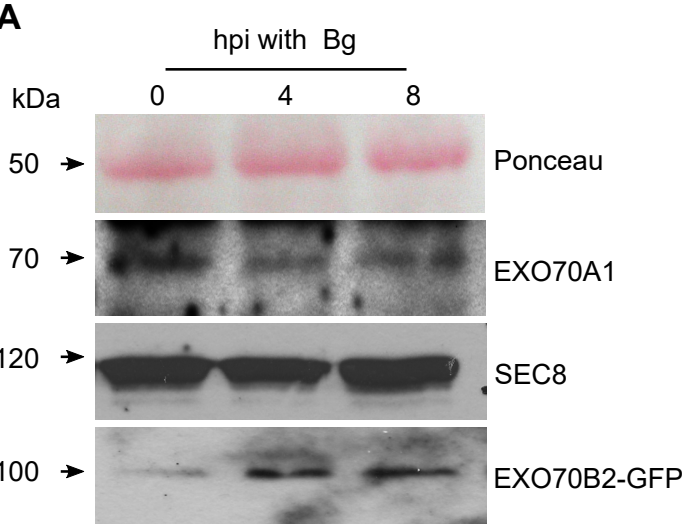

$\mathrm{kDa}$

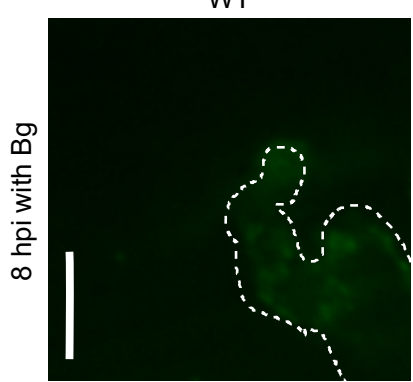

GFP-EXO70B1
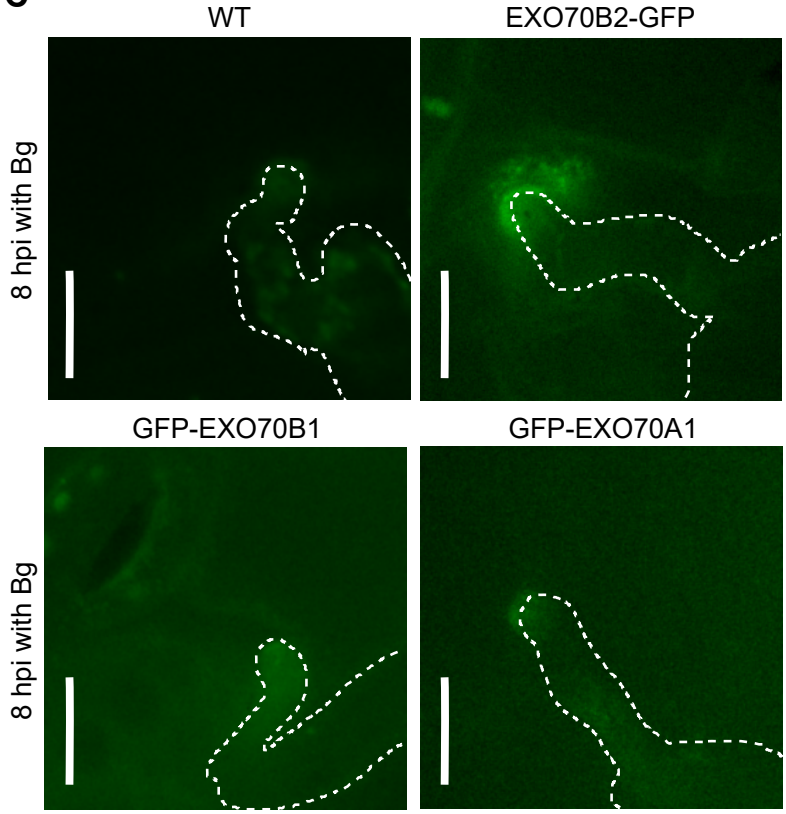

GFP-EXO70A1

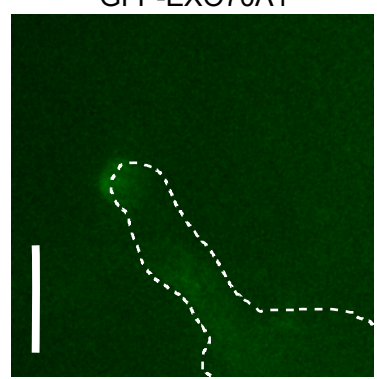

Figure 1. EXO70B2 expression dynamics after Bg inoculation. (A) Protein levels of EXO70A1, SEC8 and EXO70B2-GFP in complemented exo70B2 mutant at 0, 4, 8 hpi. (B) Protein level of GFPEXO70B1 in exo70B1 mutant at 0, 4, 8 hpi. Proteins were isolated from 4 week old plants. Anti-GFP, anti-EXO70A1 and anti-SEC8 antibodies were used. (C) The early focal accumulation of EXO70B2GFP signal in the cytoplasm in vesicle-like dots in comparison to the absent signal of GFP-EXO70B1 and faint signal of GFP-EXO70A1 at 8 hpi with Bg. All images represent a single optical section obtained by confocal spinning disc microscopy. All constructs were expressed under their natural promotors. The white dashed line indicates the fungus. The scale bar represents $10 \mu \mathrm{m}$. 

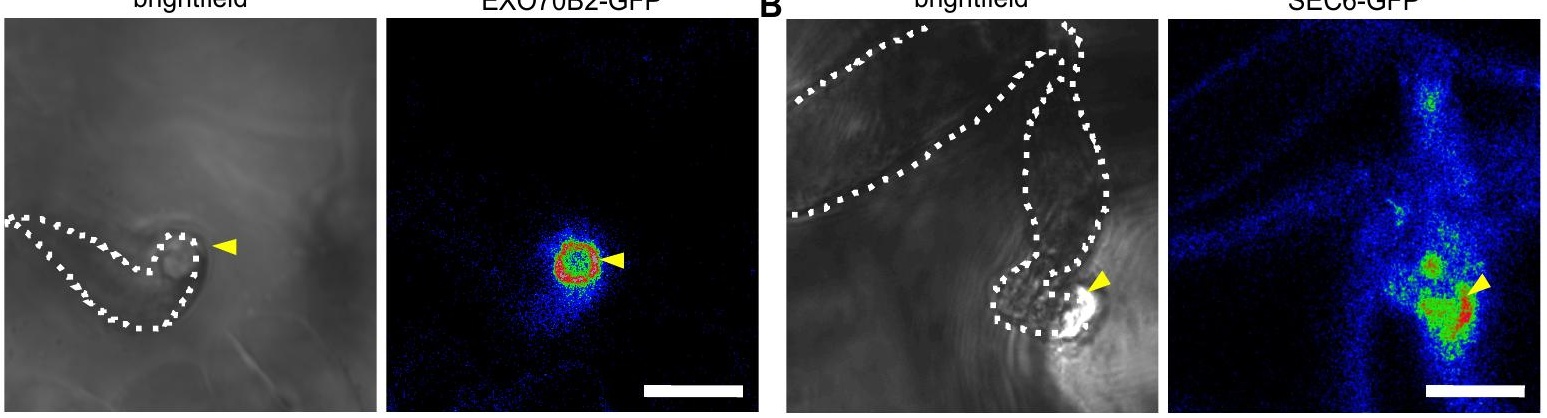

C
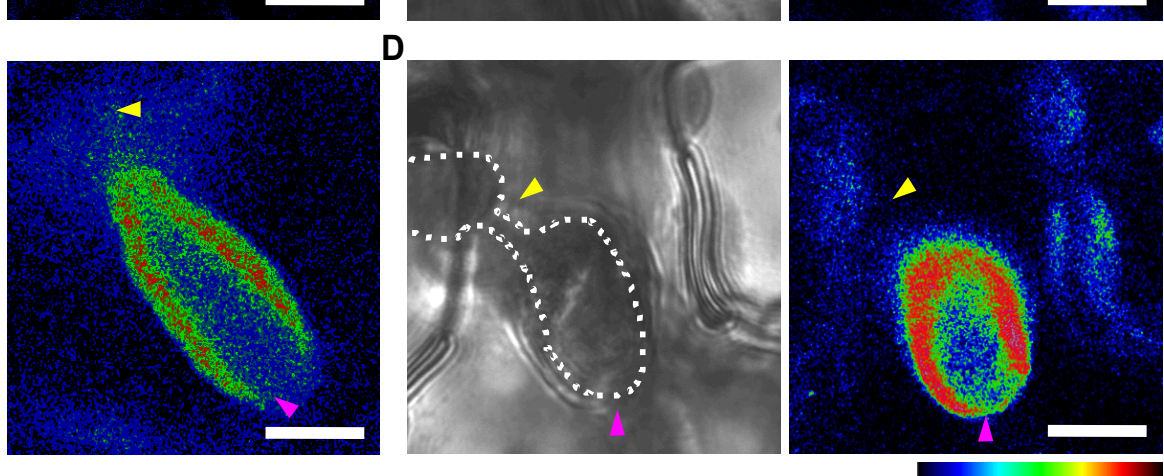

$\min$

$\max$

Figure 2. Exocyst localisation in areas of papillae and haustorial encasements. The panels represent the brightfield and signal intensity analysis of the two stages of defensive structure growth: papilla (A, B) and enclosing encasement (C, D). The EXO70B2 signal surrounds the papilla (A) and continue to the encasement (C). The SEC6GFP signal strongly marks the papilla (B) and the encasement (D). Plants were treated for 48 hours with Bg. Pink arrowheads denote the haustorium ends, where the exocyst subunit signal disappears. The white dashed line outlines fungal structures. The yellow arrowheads indicate penetration sites. All images represent a single optical section obtained by confocal microscopy. The scale bar represents $10 \mu \mathrm{m}$; the scale bar of signal intensities on the right. 

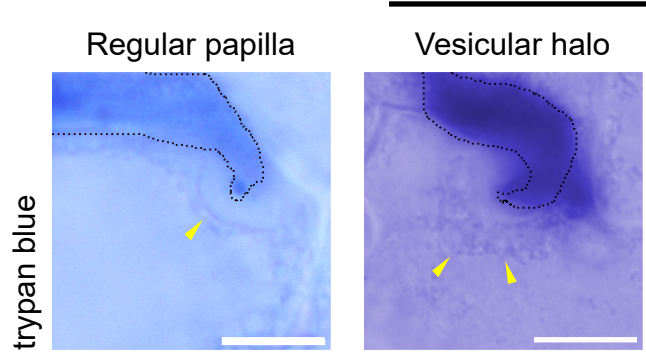

\section{Enlarged papilla}
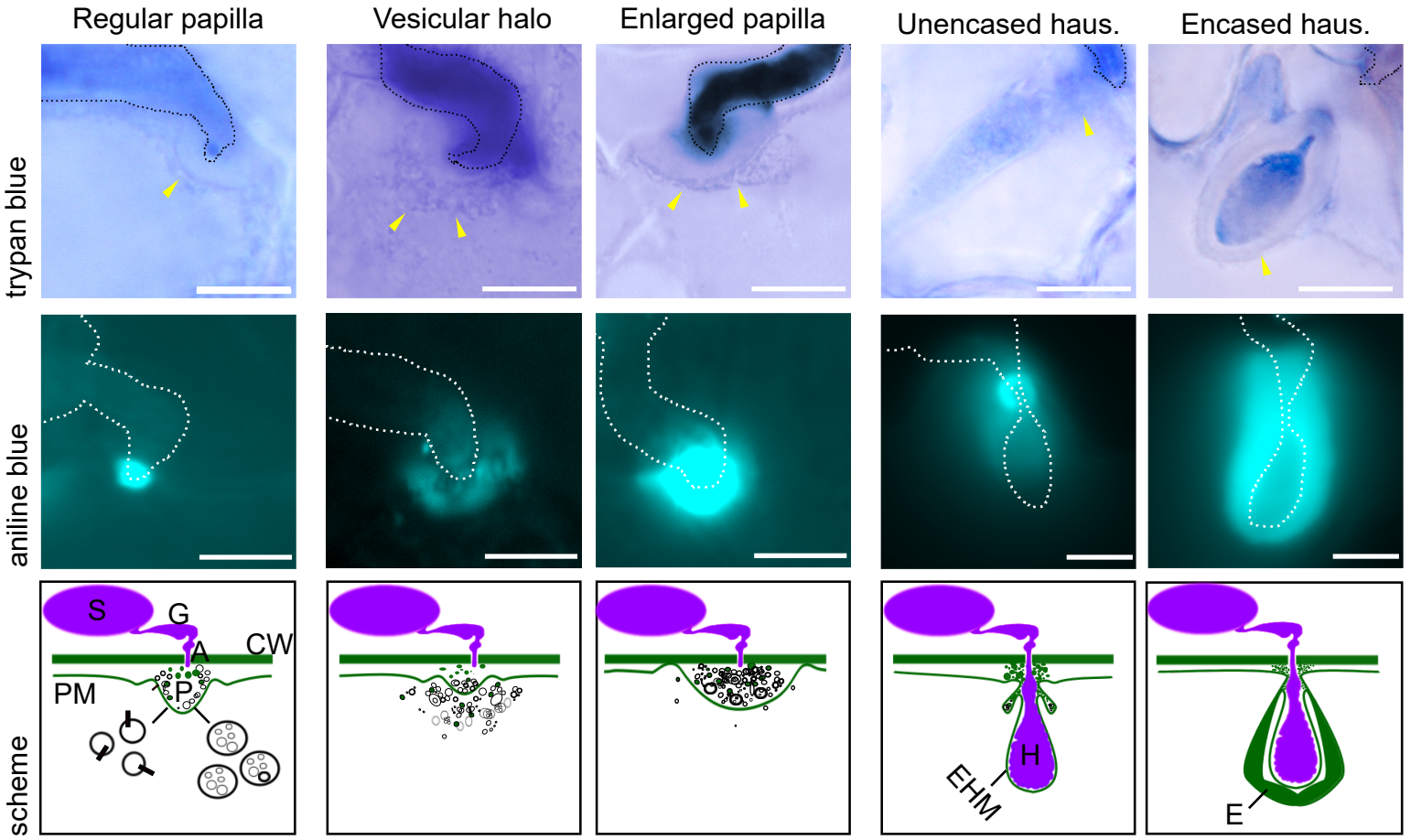

B

24 hpi Bg

48 hpi Bg
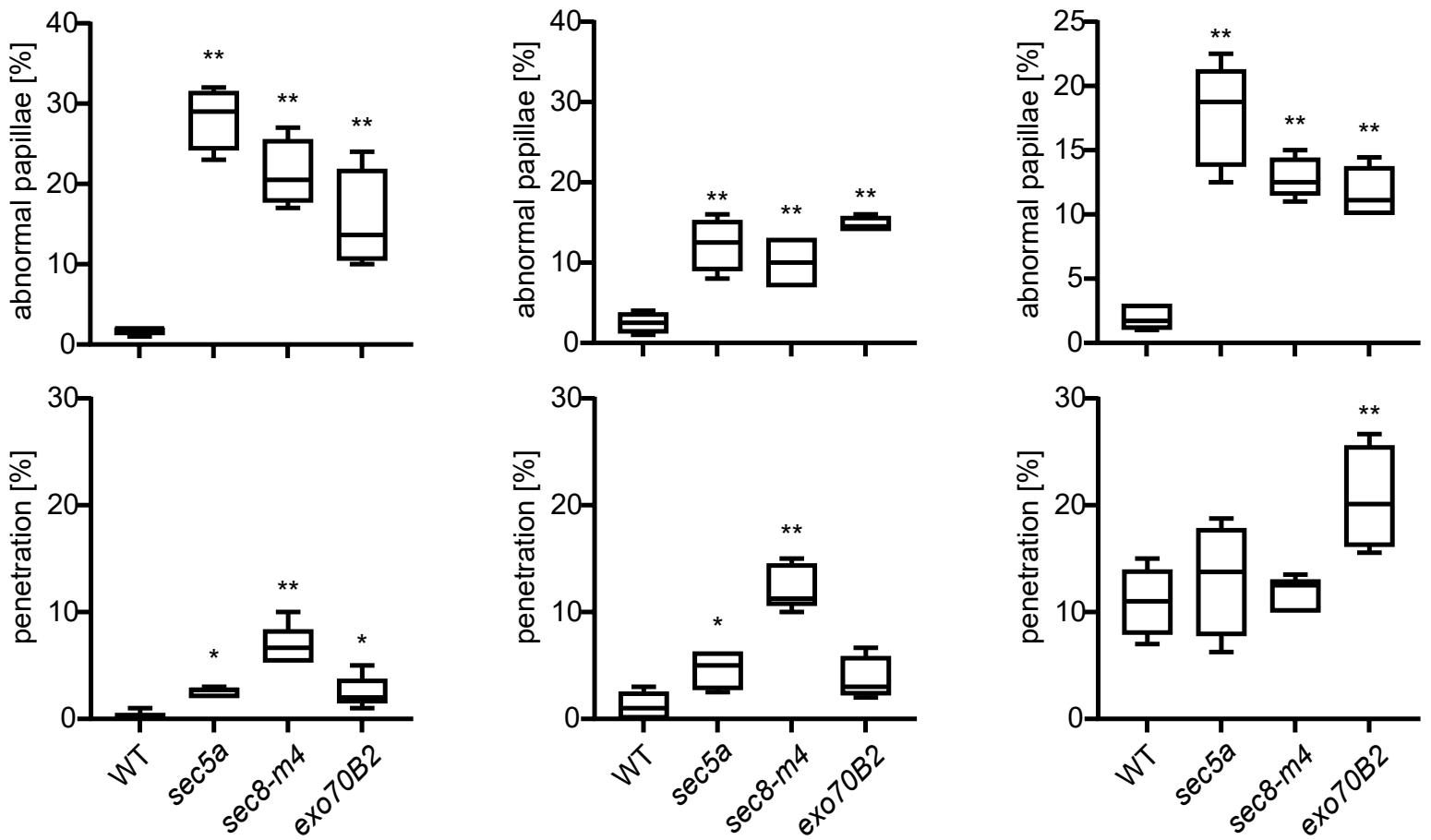

Figure 3. Category and quantification of the interactions between exocyst mutants and non-adapted pathogens. From the top: the first row shows independent $\mathrm{Bg}$ (Ep)/Arabidopsis interactions visualized by trypan blue staining; the second row displays aniline blue staining; the third row represents a schematic model of the interaction. Bg and Ep evoke the same types of interactions in Arabidopsis: regular papilla; abnormal papillae / either with a vesicular halo or enlarged papilla; penetrated cells / either unencased or encased haustorium. (B) The data show the mean of the abnormal papillae and penetrated cells by tested pathogens at different time points. For each column in the graph, the area complementary to $100 \%$ represents all interactions with spores that did not provoke any reaction of the plant. The experiment was repeated 3 times with a similar trend. In each experiment, the $\mathrm{N}$ represents 5 quantified leaves, 100 spores were counted per leaf. Stars indicate significant differences calculated by one-way ANOVA with the post-hoc Tukey-Kramer Honestly Significant Difference (HSD) test, $* *$ Z-score p $<0.01$, * $\mathrm{Z} \mathrm{p}<0.05$. For the schematic: A appressorium; GT germ tube; S spore; CW membrane; P papilla; EHM extrahaustorial membrane; H haustorium; E encasement. Yellow arrows mark outer borders of plant cell defensive structures. The dashed line outlines fungal structures. Scale bars represent $5 \mu \mathrm{m}$. 

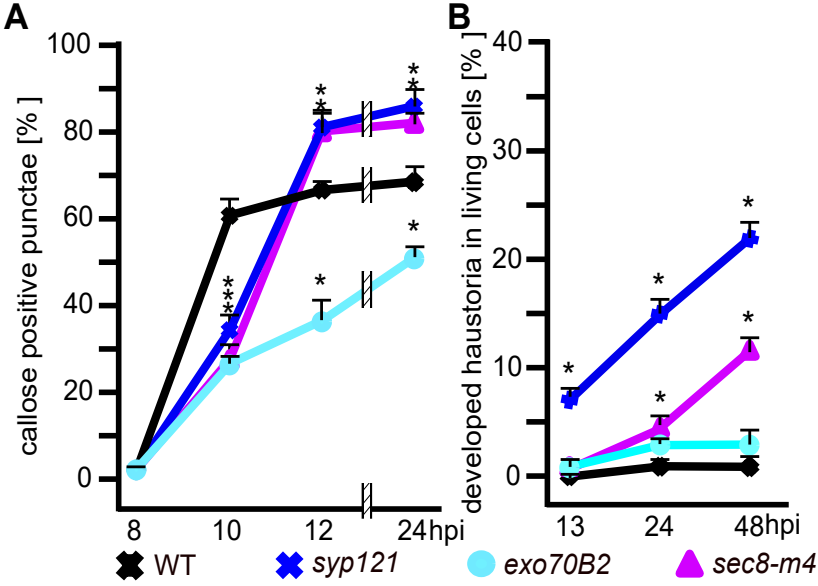

Figure 4. Effects of exocyst mutations on the occurrence of a haustorium and callose deposition in defence against Bg. (A) The frequency of callose positive punctae counted on a defined leaf area at sites of Bg attack was followed at 8, 10, 12, 24 hpi. (B) The frequency of developed haustoria in cells of WT, syp121, exo70B2 and sec8-m4 leaves. Each experiment was repeated 3 times with similar results, $\mathrm{N}$ represents 5 leaves. The asterisks indicate significant difference from WT at each time point. Statistical significance was calculated using oneway ANOVA with the post-hoc Tukey-Kramer HSD test, $Z$ score $p<0.05$. Error bars represent the standard error. 


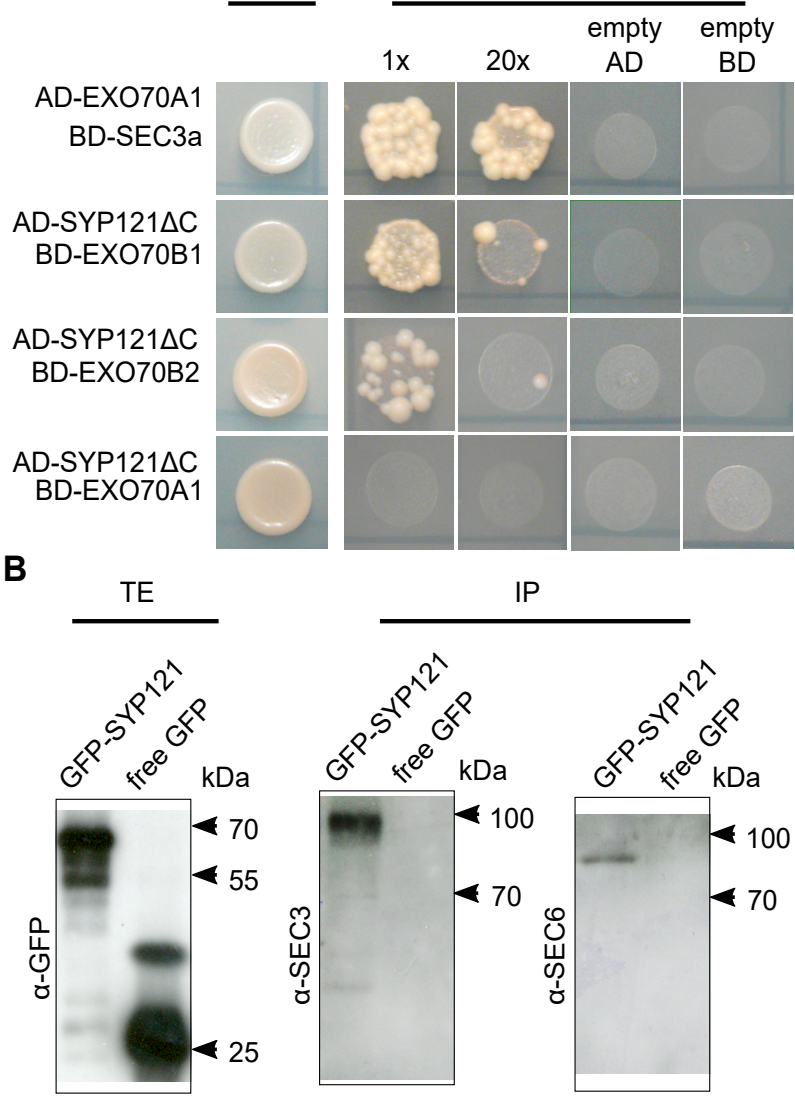

Figure 5. EXO70B2 subunit interacts with SYP121 in defence. (A) Direct interaction between the exocyst and SYP121 was examined in a yeast two-hybrid assay. Yeasts containing both the binding (BD) and activation domain (AD) were plated on the double selection SDLeu, -Trp or on the SD - His, Trp, Ade, Leu plates and allowed to grow for 6 days. For each pair, negative control transformations with empty counterpart $\mathrm{AD}$ or BD plasmids were performed. (B) The seedlings expressing GFP-SYP121 or free GFP were used for anti-GFP co-immunoprecipitation and the bound fraction was tested for the exocyst subunits presence by immunoblotting. TE; total extract, IPimmunoprecipitated proteins. 
